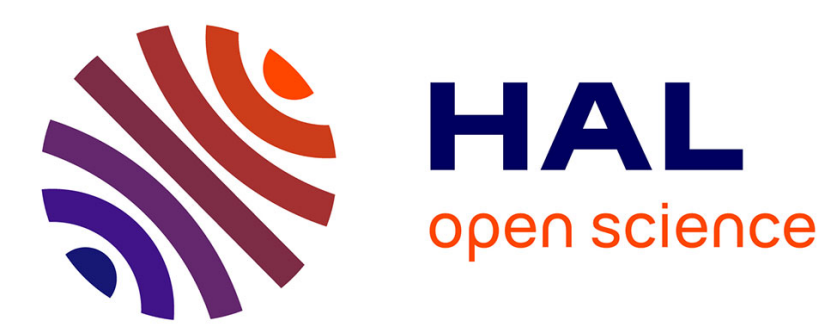

\title{
Online adaptive power allocation and channel state feedback strategies in underwater acoustic networks
}

Antony Pottier, Beatrice Tomasi

\section{To cite this version:}

Antony Pottier, Beatrice Tomasi. Online adaptive power allocation and channel state feedback strategies in underwater acoustic networks. OCEANS'21 Porto, Sep 2021, Porto, Portugal. hal-03461258

\author{
HAL Id: hal-03461258 \\ https://hal.science/hal-03461258
}

Submitted on 1 Dec 2021

HAL is a multi-disciplinary open access archive for the deposit and dissemination of scientific research documents, whether they are published or not. The documents may come from teaching and research institutions in France or abroad, or from public or private research centers.
L'archive ouverte pluridisciplinaire HAL, est destinée au dépôt et à la diffusion de documents scientifiques de niveau recherche, publiés ou non, émanant des établissements d'enseignement et de recherche français ou étrangers, des laboratoires publics ou privés. 


\title{
Online adaptive power allocation and channel state feedback strategies in underwater acoustic networks
}

\author{
Antony Pottier ${ }^{*}$ Beatrice Tomasi ${ }^{* \dagger}$ \\ *ISEN Brest - Yncrea Ouest, LabISEN \\ 20 Rue Cuirassé Bretagne, 29200 Brest, France \\ Email: \{antony.pottier,beatrice.tomasi\}@isen-ouest.yncreas.fr \\ $\dagger$ NORCE - Norwegian Research Center \\ Nygårdsgaten 112, 5008 Bergen, Norway
}

\begin{abstract}
The contribution of this paper is twofold. First we evaluate the performance of adaptive power allocations for multiuser communications in underwater acoustic channels. The study is conducted within the theoretical framework of non-cooperative games, and we consider the use of both instantaneous and statistical Channel State Information (CSI) at the transmitter side. Second, we evaluate CSI acquisition schemes to be used for adaptive power allocations strategies.

Results are obtained thanks to channels sounded at-sea at the Littoral Ocean Observatory Network (LOON) provided by the Centre for Marine Research and Experiments (CMRE), La Spezia, Italy. They show that adaptive power allocation strategies can be beneficial in some multi-user environments, even considering outdated instantaneous CSI at the transmitter side thanks to the introduction of an appropriate forgetting factor in the power allocation update algorithm.
\end{abstract}

Index Terms-Underwater acoustic communications, spectrum sharing, interference channels, channel state information, adaptive power allocation

\section{INTRODUCTION}

During the past decades, underwater acoustic communication (UAC) technologies have become more and more mature. However, the underwater acoustic (UWA) channel is still a harsh environment for establishing reliable, high rate, and robust communications. The bandwidth is very limited due to absorption and strong multi-path, and the channel varies at different time and space scales [1]-[3]. Furthermore, the underwater acoustic communication channel is not regulated, thus letting different systems interfering unwillingly with each other. Several cases of such interference situations have been reported in the literature [4], [5].

This makes both point-to-point and multi-user communications a challenging task and future developments call for adaptive modems and networks [6]. However, the feedback on channel information from the receiver to the transmitter is usually needed to allow an autonomous adaptation of the transmission strategies to the environment (including the channel and interference from other possible users).

The contribution of this paper lies on:

1) The performance analysis of the adaptive power allocation strategies through experimental data, in a multi-user, non-cooperative context. Two non-cooperative spectrum allocation games for underwater acoustic interference channels with their solution will be formulated. The solutions of this game allow to implement an adaptive power allocation scheme that is totally decentralized and non-cooperative, based only on the local information each TX is able to obtain from its RX.

2) A comparative experimental analysis of CSI acquisition strategies. The experimental results will provide guidelines on how to design CSI acquisition and algorithms for adaptive power allocation. Instantaneous and statistical Channel State Information (CSI) at the transmitter side will be considered. The theoretical performance achieved in terms of information rates will be given for the two types of CSI. These comparisons constitutes an extension of the works in [7], [8].

Notations: Upper case bold letters $\boldsymbol{A}$ denotes matrices, while lowercase bold letters $\boldsymbol{x}$ are used for vectors. The $n^{\text {th }}$ coordinate of a vector $\boldsymbol{x}$ is written $(\boldsymbol{x})_{n}$ and the coefficient at line $n$ and column $m$ of matrix $\boldsymbol{A}$ is denoted by $[\boldsymbol{A}]_{n m}$. Sets of integers of the form $\{1, \cdots, N\}$ are written $\llbracket 1, N \rrbracket$. Uppercase letters $X$ denotes random variables or (discrete time) processes, and $x$ or $x[t]$ denote their samples (or realizations). Finally, $\mathbb{E}$ stands for expectations.

\section{SYSTEM MODEL}

We consider $I=2$ transmitter-receiver (TX-RX) couples in an interference channel. The channel is divided in $N$ sub-channels (considering a modulation such as orthogonal frequency division multiplexing (OFDM)). Each transmitter aims at finding the power allocation strategy maximizing an utility function $u_{i}$ related to its information rate, considering the interference from other users as additive noise. The power allocation strategy must be chosen by the transmitter on the basis of a CSI fed back from its intended receiver.

Let $\boldsymbol{x}_{i}[t]=\left(x_{i, 1}[t], \cdots, x_{i, N}[t]\right)^{T}$ be the vector of zeromean i.i.d. complex symbols sent by TX $i$ at time $t$ on the $N$ available sub-channels. The bandwidth of each sub-channel is given by $\Delta_{f}=B / N$, and the symbol duration is $T$, including eventual guard intervals. For any $j \neq i$ the symbols $\boldsymbol{x}_{j}[t]$ are independent of $\boldsymbol{x}_{i}[t]$. Each TX has the same power constraint

$$
\sum_{n=1}^{N} p_{i, n} \leq P_{\max }
$$


where $p_{i, n}=\mathbb{E}\left[\left|X_{i, n}\right|^{2}\right]$ is the power allocated by TX $i$ on the $n^{\text {th }}$ sub-channel.

The distance separating $\mathrm{RX} i$ from $\mathrm{TX} j$ induces a propagation delay $\Delta_{j i}$. Neglecting inter-carrier interference (ICI) and considering that the receiver is correctly synchronized, after demodulation and Fast Fourier Transform, the channel output for the receiver $i$ at time $t$ is given by

$$
\boldsymbol{y}_{i}[t]=\boldsymbol{H}_{i i}[t] \boldsymbol{x}_{i}\left[t-\Delta_{i i}\right]+\boldsymbol{H}_{j i}[t] \boldsymbol{x}_{j}\left[t-\Delta_{j i}\right]+\boldsymbol{w}_{i}[t]
$$

where $\boldsymbol{x}_{j}[t]$ are the symbols sent by TX $j$ at time $t$, independent of those from TX $i ; \boldsymbol{H}_{j i}[t]$ are $N \times N$ diagonal matrices where $h_{j i, n}[t]=\left[\boldsymbol{H}_{j i}[t]\right]_{n n}, n=1, \cdots, N$, is the channel sample at frequency $n$ and time $t$ between $\operatorname{TX~} j$ and $\mathrm{RX} i$. These channel coefficients are issued from a stationary random process $H_{j i, n}$. Finally $\boldsymbol{w}_{i}[t]$ are Gaussian noise samples, with $W_{i, n} \sim \mathcal{C N} \mathcal{N}\left(0, \sigma_{i, n}^{2}\right)$. The random variables of the symbols $X_{j, n}$ and $X_{i, n}$, the noise $W_{i, n}$ and the channels $H_{j i, n}$ and $H_{i i, n}$ are independent.

\section{Problem Statement AS A NON-COOPERATIVE GAME}

Following the works of [9], we formulate the problem as a non-cooperative game

$$
\mathcal{G}=\left(I,\left(\mathcal{A}_{i}\right)_{i=1}^{I},\left(u_{i}\right)_{i=1}^{I}\right)
$$

where

- The $I$ TX-RX pairs are the players of the game,

- $\mathcal{P}_{i}=\left\{\boldsymbol{p}_{i} \in \mathbb{R}^{N},\left\|\boldsymbol{p}_{i}\right\|_{1} \leq P_{\max }\right\}$ is the action set of the player (TX) $i$, corresponding to the feasible power allocation vectors on $N$ sub-channels under a power constraint.

- $u_{i}$ is the utility function to be maximized, and is related to the information rate of user $i$.

The standard solution of a non-cooperative game is given by the Nash Equilibrium (NE) concept, whose definition is recalled below :

Definition 1. A Nash Equilibrium of the game $\mathcal{G}$ is an action profile $\left(\boldsymbol{p}_{i}, \boldsymbol{p}_{j}\right)$ such that for all player $i$ we have

$$
u_{i}\left(\boldsymbol{p}_{i}, \boldsymbol{p}_{j}\right) \geq u_{i}\left(\boldsymbol{p}_{i}^{\prime}, \boldsymbol{p}_{j}\right) \quad \forall \boldsymbol{p}_{i}^{\prime} \in \mathcal{P}_{i}
$$

Alternatively, a NE can be defined thanks to the best responses $(\mathrm{BR})$ of the players :

Definition 2. $A$ best response (BR) of player $i$ to a fixed opponents action $\boldsymbol{p}_{j}$ is any action in the set

$$
B R_{i}\left(\boldsymbol{p}_{j}\right)=\left\{\boldsymbol{p}_{i} \in \mathcal{P}_{i}, u_{i}\left(\boldsymbol{p}_{i}, \boldsymbol{p}_{j}\right) \geq u_{i}\left(\boldsymbol{p}_{i}^{\prime}, \boldsymbol{p}_{j}\right) \forall \boldsymbol{p}_{i}^{\prime} \in \mathcal{P}_{i}\right\},
$$

which possibly reduces to a singleton when $u_{i}$ is concave in $\boldsymbol{p}_{i}$.

According to this concept, a NE is an action profile such that every player best-responds to its opponents. This interpretation of a NE justifies the use of the Best Response Dynamics (BRD) algorithm [10], [11] to compute the NEs of the games we will formulate in this paper. Note that at least one NE exists in every game with compact and convex actions sets and utility functions $u_{i}\left(\boldsymbol{p}_{i}, \boldsymbol{p}_{j}\right)$ concave in $\boldsymbol{p}_{i}$. This is verified by the sets $\mathcal{P}_{i}$ and the utility functions defined in the next subsections.

Two different utility functions will be defined depending on the nature of the CSI fed back by the RXs to their intended TXs. The two considered situations are the following : the RXs send back 1) an instantaneous CSI assumed to be obtained thanks to pilot symbols and fed back at the TXs side with some propagation delays, 2) a statistical CSI obtained as a function of channels statistics assumed to be known. Equation (9) defines the utility function for the first game with instantaneous CSI, while equation (14) corresponds to the game with statistical CSI. Note that these utility functions are metrics guiding the TXs in their choice of power allocation strategies, and performance of these strategies will be evaluated in terms of information rates defined in equations (6) and (13). By maximizing this utility function, the TXs (the players of the game) plays actually their BR to the power allocation of the other channel users. This BR takes the form of a water-filling (WF) allocation over the noise plus interference PSD weighted by the inverse of the direct channel gains. The BRD consists, for the players, to play their BR one after the other until convergence to a NE of the game (see [7], [12], [13] for details on the technical conditions). In the present context of UWA communication in an interference channel, the players need only to know their direct channel gain and the aggregate PSD of the noise plus interference to update their own power allocation strategy by WF. One of the main advantage of such solutions is that it can be implemented in a totally decentralized and non-cooperative way, with no coordination or information exchanges between the users. Details are given in the two next subsections.

\section{A. Game with feedback of instantaneous CSI}

Assume here that, at time $t, \operatorname{RX} i$ knows its sub-channels coefficients $h_{i i, n}[t]$ and the noise plus interference power ${ }^{1}$. At time $t, \mathrm{RX} i$ receives a new observation vector $\boldsymbol{y}_{i}[t]$ and feeds back a CSI to its corresponding TX, which updates its power allocation. The power allocated by $\mathrm{TX} j$ at time $t$ is perceived at time $t+\Delta_{j i}$ at $\mathrm{RX} i$ because of the propagation delay. We denote by $p_{j, n}[t]$ the power that TX $j$ allocates on the subchannel $n$. The "instantaneous rate" of user $i$ at time $t$ is then given (in bits/s/Hz) by

$$
R_{i}[t]=\alpha \sum_{n} \log _{2}\left(1+\gamma_{i, n}[t] p_{i, n}\left[t-\Delta_{i i}\right]\right)
$$

where $\alpha=\left(N T \Delta_{f}\right)^{-1}$ and the CSI at the RX $i$ is given by

$$
\gamma_{i, n}[t]=\frac{\left|h_{i i, n}[t]\right|^{2}}{\sigma_{i, n}^{2}+\left|h_{j i, n}[t]\right|^{2} p_{j, n}\left[t-\Delta_{j i}\right]} .
$$

The instantaneous CSI undergoes a delay $\Delta_{i i}$ (in number of blocks of $N$ symbols) when available at TX $i$, as a consequence of the low propagation speed of the acoustic wave $(\approx 1500 \mathrm{~m} / \mathrm{s})$. The CSI at TX $i$ is then defined by

$$
\gamma_{i, n}^{\text {inst }}[t]=\frac{\left|h_{i i, n}\left[t-\Delta_{i i}\right]\right|^{2}}{\sigma_{i, n}^{2}+\left|h_{j i, n}\left[t-\Delta_{i i}\right]\right|^{2} p_{j, n}\left[t-\Delta_{j i}-\Delta_{i i}\right]} .
$$

${ }^{1}$ The channel can be estimated thanks to pilots symbols inserted in the data. For the noise and interference power estimation, we refer the reader to [14] for an example applying to adaptive OFDM. 
TX $i$ optimizes its power allocation vector according to the CSI obtained from its RX. The problem can be formulated as utility maximization with a utility function $u_{i}^{\text {inst }}$ defined by

$$
u_{i}^{\text {inst }}\left(\boldsymbol{p}_{i}, \boldsymbol{p}_{j}\right)=\alpha \sum_{n} \log _{2}\left(1+\gamma_{i, n}^{\text {inst }}[t] p_{i, n}\right) .
$$

This function is maximized by a unique power allocation vector given by the WF operator, which is, consequently, the best response of TX $i$ against the power allocation of the others users (perceived through the delayed CSI). At time $t, \mathrm{TX} i$ updates its power allocation strategy $\boldsymbol{p}_{i}$ as follows :

$$
\boldsymbol{p}_{i}[t+1]=W F_{i}\left[\boldsymbol{\gamma}_{i}^{\text {inst }}[t]\right]
$$

where $\gamma_{i}^{\text {inst }}[t]=\left(\gamma_{i, 1}^{\text {inst }}[t], \cdots, \gamma_{i, N}^{\text {inst }}[t]\right)$, and $\forall n \in \llbracket 1, N \rrbracket$

$$
p_{i, n}[t+1]=\left(W F_{i}\left[\gamma_{i}^{\text {inst }}[t]\right]\right)_{n}=\max \left(\lambda_{i}-\frac{1}{\gamma_{i, n}^{\text {inst }}[t]}, 0\right)
$$

where the water-level $\lambda_{i}$ is chosen to satisfy the power constraint of equation (1). As the WF allocation is the BR of a player to the allocation of the other user, applying an iterative WF scheme distributed among the players is equivalent to the BRD in game theory, where players best respond to each other. In this game we will consider a "smoothed" version of the algorithm [13] by introducing a forgetting factor $\beta \in[0,1$ (, thus replacing the update of (11) by

$$
\boldsymbol{p}_{i}[t+1]=\beta \boldsymbol{p}_{i}[t]+(1-\beta) W F_{i}\left[\boldsymbol{\gamma}_{i}^{\text {inst }}[t]\right] .
$$

As we will see in the results section, this forgetting factor can help to track the channels variations in time as well as to cope with slightly outdated CSI. Algorithm 1 sums up the procedure.

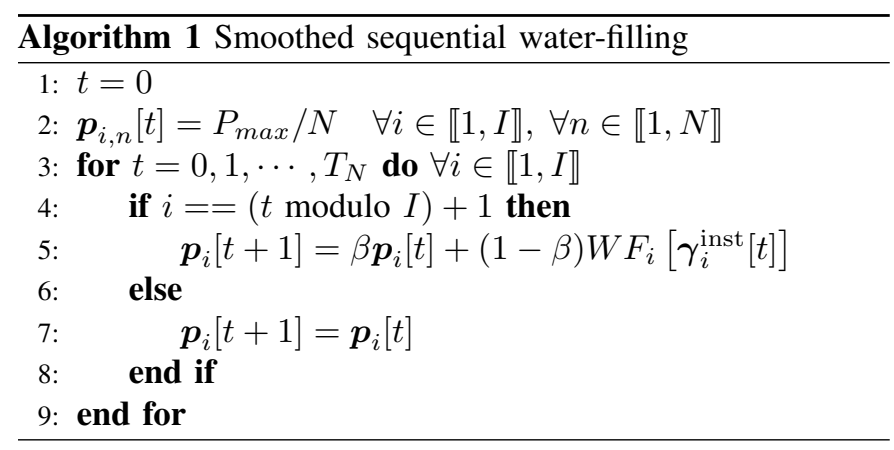

\section{B. Game with feedback of statistical CSI}

Assuming that the coherence time of the channels is sufficiently small regarding $L \times T$, the transmitters will try to optimize a utility function related to their average information rate over $L$ symbols

$$
\bar{R}_{i}=\alpha \sum_{n=1}^{N} \mathbb{E}\left\{\log \left(1+\frac{\left|H_{i i, n}\right|^{2} p_{i, n}}{\sigma_{i, n}^{2}+\left|H_{j i, n}\right|^{2} p_{j, n}}\right)\right\}
$$

given in bits/s/Hz for fixed power allocations $\boldsymbol{p}_{i}$ and $\boldsymbol{p}_{j}$. Expectations are taken relatively to the channels distributions.

Maximizing (13) requires the TXs to know either the channels distributions or their realizations. Here, it is assumed that the channel coherence time prohibits feedback of an instantaneous CSI (this is the case when the transmission range is such that the propagation delays is significantly higher than the coherence time). We adopt the robust approach in [7], [9] where the utility functions are defined as the worst ergodic rate relatively to the interference distributions. We have

$$
\bar{u}_{i}\left(\boldsymbol{p}_{i}, \boldsymbol{p}_{j}\right)=\alpha \sum_{n} \log _{2}\left(1+\gamma_{i, n} p_{i, n}\right)
$$

with the statistical CSI given by

$$
\gamma_{i, n}=\frac{\mathrm{e}^{\mathbb{E}\left[\log \left|H_{i i, n}\right|^{2}\right]}}{\sigma_{i, n}^{2}+\mathbb{E}\left[\left|H_{j i, n}\right|^{2} p_{j, n}\right]} .
$$

This statistical CSI is assumed known by the transmitter. Considering stationary channels, it can be considered that, prior to each transmission of $L$ symbols, the RX compute the statistics of the channels and interference plus noise power spectral density (PSD) ${ }^{2}$.

In this game the BR of a player to the power allocation of its opponent is also a WF solution, but computed here thanks to the statistical CSI available at the TX :

$$
\boldsymbol{p}_{i}^{\star}=W F_{i}\left[\gamma_{i}\right]
$$

where $\gamma_{i}=\left(\gamma_{i, 1}, \cdots, \gamma_{i, N}\right)^{T}$ and $\forall n \in \llbracket 1, N \rrbracket$

$$
\left(W F_{i}\left[\gamma_{i}\right]\right)_{n}=\max \left(\lambda_{i}-\frac{1}{\gamma_{i, n}}, 0\right)
$$

with $\lambda_{i}$ chosen to satisfy the power constraint (1). As previously, an instance of the BRD can be implemented by players playing the WF power allocation against each other, based on their own statistical CSI. This corresponds to Algorithm 1 with $\beta=0$ and replacing $\gamma_{i}^{\text {inst }}[t]$ by $\gamma_{i}$. Another difference with the previous algorithm lies in how the power allocation updates are scheduled : here, each receiver needs to wait enough time after each update in order to compute the average interference PSD, while in Algoritm 1 each transmitter updates its strategy when the instantaneous CSI is available.

\section{EXPERIMENTAL EVALUATION OF ADAPTIVE POWER ALLOCATION STRATEGIES}

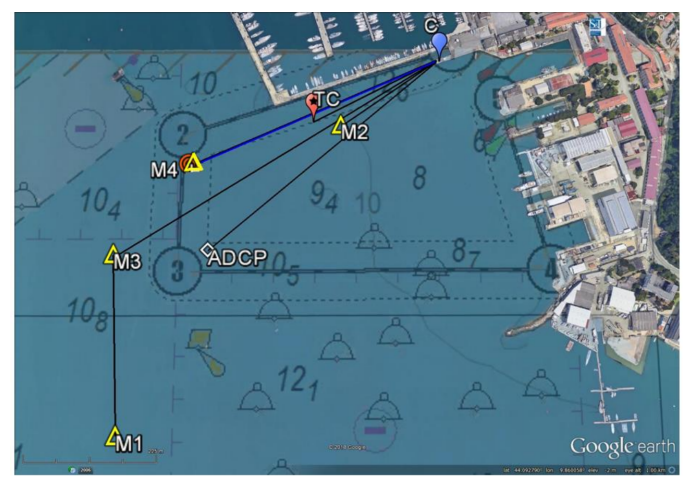

Fig. 1. Experiment set-up at the LOON testbed.

\footnotetext{
${ }^{2}$ The interference PSD may change with time but we can neglect this fact by considering that the transmissions of the two users start approximately at the same time in order to simplify the presentation.
} 
TABLE I

RELATIVE DISTANCES BETWEEN TRIPODS OF THE LOON TESTBED.

\begin{tabular}{|c|c|c|c|c|}
\hline & M1 & M2 & M3 & M4 \\
\hline M1 & - & $660 \mathrm{~m}$ & $310 \mathrm{~m}$ & $480 \mathrm{~m}$ \\
\hline M2 & $660 \mathrm{~m}$ & - & $460 \mathrm{~m}$ & $280 \mathrm{~m}$ \\
\hline M3 & $310 \mathrm{~m}$ & $460 \mathrm{~m}$ & - & $200 \mathrm{~m}$ \\
\hline M4 & $480 \mathrm{~m}$ & $280 \mathrm{~m}$ & $200 \mathrm{~m}$ & - \\
\hline
\end{tabular}
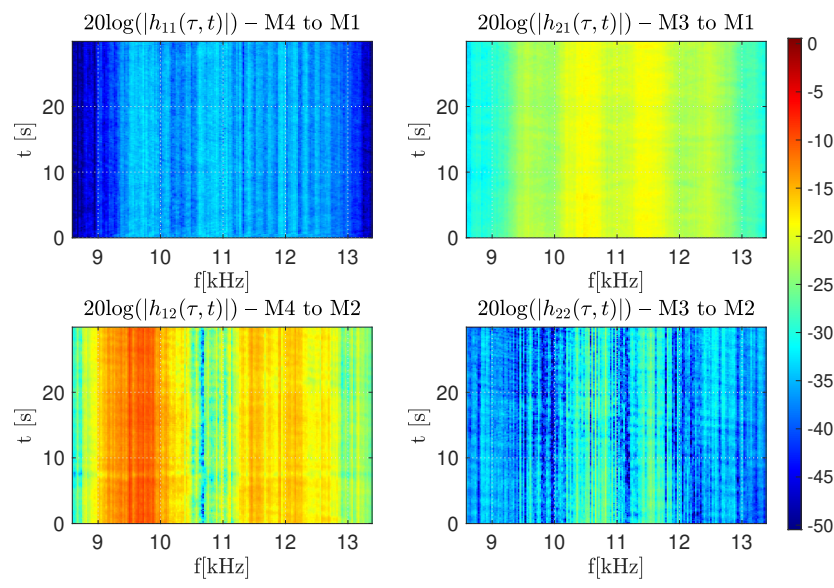

Fig. 2. Time varying frequency responses used for the power allocation games.

The experimental set up, provided by STO NATO CMRE, is represented in Figure 1. It consisted of four tripods deployed in the sea bottom in La Spezia harbor (Italy). Tripods M1, M2 and M3 were equipped with an acoustic source and an hydrophone, while M4 is equipped only with an acoustic source. Relative distances between nodes are summarized in Table I.

The channels between the different tripods were sounded to simulate an interference channel with $I=2$ users on the basis of real data. Soundings were performed by matched filtering to a known probe signal made of 255 BPSK symbols modulated at a 4.8 kbauds modulation speed centered on $f_{0}=11 \mathrm{kHz}$. The delay spread covered is $\tau_{D}=53.1 \mathrm{~ms}$, the bandwidth $B_{s}=5.280 \mathrm{kHz}$, and 564 such probes were sent continuously during an observation time $T_{o b s}=30 \mathrm{sec}$. Figure 2 shows the time varying frequency responses of the four channels used for the 2-users interference channel.

The two users correspond respectively to the links M4 to M1 and M3 to M2 (TXs are M4 and M3). This is a relatively strong interference scenario as can be seen from the channel responses, since the channel gain is on average higher on the cross-talk channels than on the direct channel. The two TXRX links are the players of non-cooperative power allocation games. The TXs use the same number of sub-channels $N=$ 180 on the same bandwidth $B=4.8 \mathrm{kHz}$. The carrier spacing is $\Delta_{f}=26.67 \mathrm{~Hz}$, and $T_{s}=1 / \Delta_{f}=37.5 \mathrm{~ms}$. Adding a guard time $T_{g}=15.6 \mathrm{~ms}$ results in a symbol duration $T=$ $T_{s}+T_{g}=53.1 \mathrm{~ms}$ (which corresponds to the delay span of the sounded channels, so that one channel sample correspond to one block of $N$ symbols). The acoustic noise power at the RXs is simulated using Knudsen's approximation [15] of a linear PSD on the logarithmic frequency scale $: \sigma^{2}(f)=$
$B_{0}-18 \log _{10}(f)$. The noise variance is scaled to achieve an average Signal to Noise Ratio (SNR) of $20 \mathrm{~dB}$ for the worst direct channel.

\section{A. Game with instantaneous CSI}

We consider that the RXs obtain the channel gain and the interference plus noise power from each received symbol at time $t$ to form the instantaneous CSI $\left(\gamma_{i, n}[t]\right)_{n=1}^{N}$ of equation (7). At the same time, TX $i$ receives from its RX the CSI delayed by a number of symbols $\Delta_{i i}$ corresponding (approximately) to the distance between TX $i$ and $\mathrm{RX} i$ assuming an acoustic wave propagating at $c=1500 \mathrm{~m} / \mathrm{s}$. All the delays mentioned in Section III-A are taken into account similarly. On the basis of its CSI, TX $i$ updates its power allocation in a proportion given by the forgetting factor $\beta$. Only one TX plays per time $t$, and they play one after the other, thus implementing Algorithm 1 starting from the uniform power allocation.

Figure 3 shows the evolution in time of the sum "instantaneous rate" of equation (6) (after summing up for the 2 users), as the Algorithm 1 is run for each channel sample, and for several values of the forgetting factor $\beta$. We can see that for $\beta=0$, when the players do not take past allocations into account, the performance curve describes cycles. These are a consequence of both the channels and interference varying in time and the outdated CSI due to propagation delays. When the forgetting factor is set to 0.3 and 0.6 it can be seen that, after 5 seconds, the power allocation strategies chosen by the users achieve a significantly higher performance than at the initial step of uniform power allocation. Furthermore, the algorithm seems to be able to track the channel variations in some extent. The benefit of applying a forgetting factor in the algorithm is clear, as it allows the players to take portion of the past history of play into account to cope with time-varying environment and outdated CSI. However, in the considered channels the propagation delays are only of few symbols $\left(\Delta_{11} \approx \Delta_{22} \approx 6\right.$ symbols and $\Delta_{21} \approx \Delta_{12} \approx 4$ symbols) due to the relatively close distances between the four nodes in the test-bed. We expect the algorithm based on instantaneous CSI to be less and less efficient as the propagation delays increase. As usual, there is also an obvious compromise between the amount of past iterations taken into account for the next updates of the algorithm and its convergence time, as can be clearly seen from Figure 3.

Power allocation strategies averaged over the 10 last iterations of the game are shown in Figure 4-(top), where it can be seen that the players have autonomously reached an operating point close to an FDMA allocation. This is due to the strong interference that lead the users to transmit on separate frequency bands. On the contrary, as shown in bottom panel of Figure 4, the power allocations strategies of the two users are closer to the initial uniform allocation when the interference is weak. In this case, the interference does not create an incentive for the players to "orthogonalize" their spectrum usage and they behave almost as if they were two separate single users in their direct channel. A plot of the performance in terms of information rate would show no significant improvement 


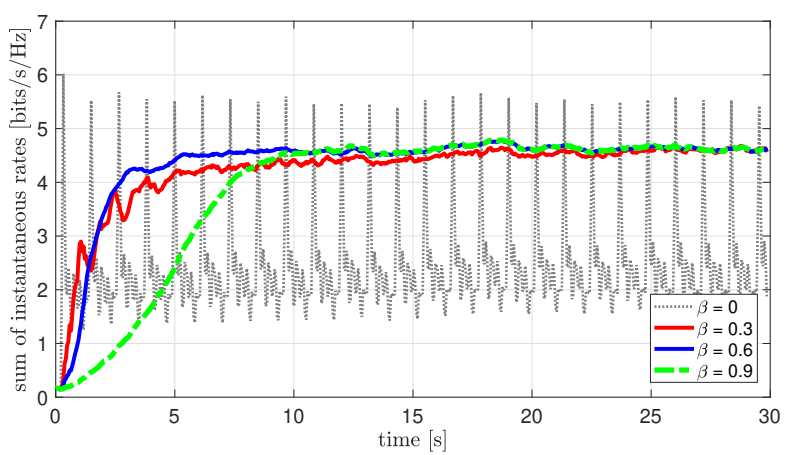

Fig. 3. Sum of "instantaneous rates" of equation (6) for the 2 users interference channels of Figure 2 and power allocations computed through Algorithm 1 at each channel sample.
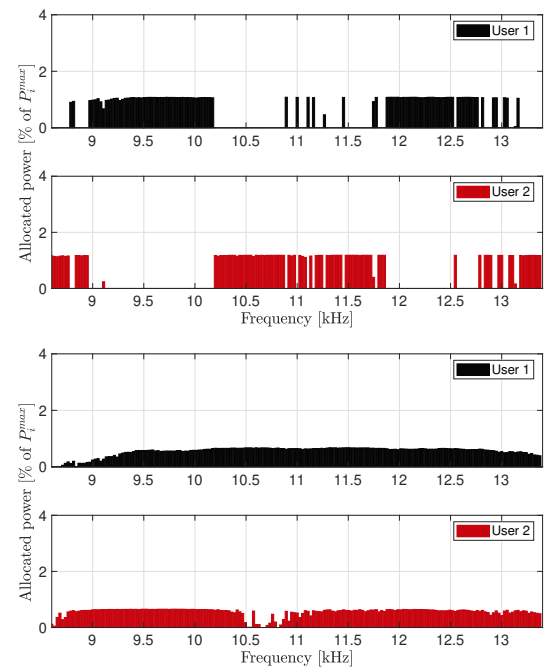

Fig. 4. Averaged power allocation of the two users over the last 10 iterations of the Algorithm 1 with $\beta=0.3$. Top: Game with strong interference and channels from Figure 2. Bottom: Game with weak interference where the role of nodes M1 and M3 are reversed compared to Figure 2.

compared to the uniform allocation (see for example [7] for similar results. This is also an echo of the work of Aval et al. on power allocation strategies for single user OFDM systems in underwater acoustic channels [16]).

It must be mentioned that, technically, the strategy profiles shown here are not NE of the game with instantaneous CSI since the inputs of this game change at each iteration of the algorithm, due to the random variations of the channels gains. This game can in fact not be solved using simple tools from non-cooperative game theory (unlike the game with statistical CSI) and would require a more advanced modeling work ${ }^{3}$.

\section{B. Game with statistical CSI}

Here, it is considered that the RXs aggregate the channels and noise plus interference PSD during a whole period of observation of the channel $\left(T_{o b s}=30 \mathrm{sec}\right)$ before sending the resulting CSI to their TXs. This must be repeated at each iteration of the distributed WF algorithm in order to provide

\footnotetext{
${ }^{3}$ Using stochastic games, as an example. This is beyond the scope of this paper and will be addressed in future works on the topic.
}

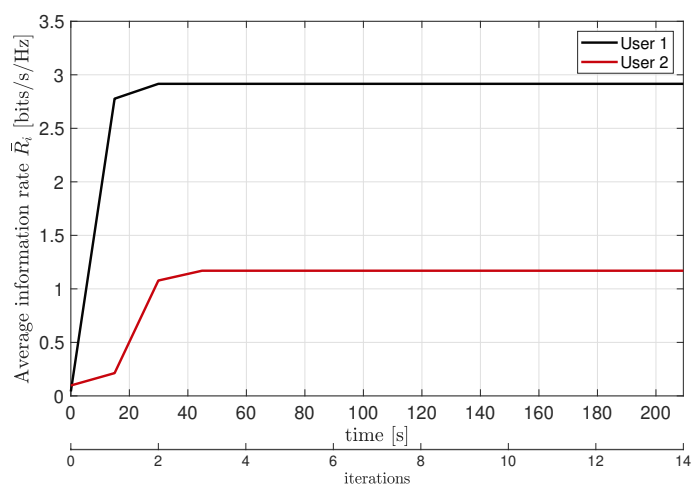

Fig. 5. Average information rates in the game with statistical CSI.

TX $i$ with the knowledge of the statistical CSI $\gamma_{i, n}$ (15), since the interference PSD is also updated at each iteration. The channels are implicitly assumed to be stationary and the expectations of equation (15) are computed by temporal averages of the realizations of the channels in Figure 2. The inputs of the game are then fixed and the distributed WF algorithm based on the channel statistics can thus converge to a NE, starting from a uniform power allocation for both players.

The results of this game are depicted in Figure 5 in terms of average information rate for each user. It can be seen that the two users converge to the $\mathrm{NE}$ of the game after few iterations. However, since they need a whole observation duration between iterations, this corresponds to a significantly higher convergence time than in Algorithm 1. As a counterpart, the feedback activity of the receivers is considerably reduced.

Two interesting things can be noticed from the NE power allocations in Figure 6. First, in the strong interference scenario, user 2 allocates more power on fewer sub-channels than in the game with instantaneous CSI. This can be explained as follows: in the game with instantaneous CSI, user 2 may benefit from the channels fading allowing it allocate its power on sub-channels that exhibit high gains on some iterations of the game but have lower gain in the average over the whole observation time. This influences also the behavior of user 1 , which "remembers" that user 2 used these channels thanks to the forgetting factor. Thus, user 2 somehow preempts these sub-channels, so that user 1 has no more incentive to use these because of the interference it experiences. This interpretation is also confirmed with Figure 7 which depicts the "instantaneous rate" (6) of both users using Algorithm 1 (in plain lines) and using the NE power allocation of the game with statistical CSI (in dotted lines). It can be seen that user 2 benefits from its allocation on a wider frequency band when it uses its instantaneous CSI, to the detriment of user 1 which undergoes extra interference which decreases its performance compared to the NE allocation in the game with statistical CSI. Second, in the weak interference configuration, we see that the $\mathrm{NE}$ allocation strategy is similar to the averaged allocation in the last steps of the game with instantaneous CSI. Since the interference has only little influence on the behavior of the 

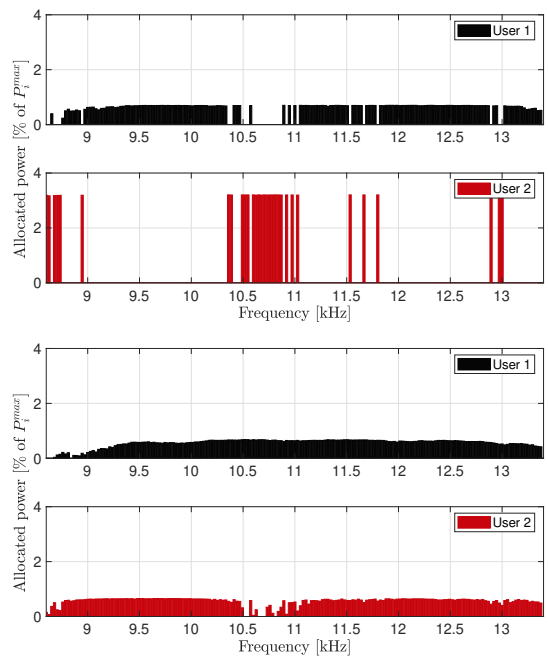

Fig. 6. Power allocation profile at the Nash Equilibrium of the game with statistical CSI. Top: strong interference with the channels of Figure 2. Bottom: weak interference where the role of nodes M1 and M3 are reversed.

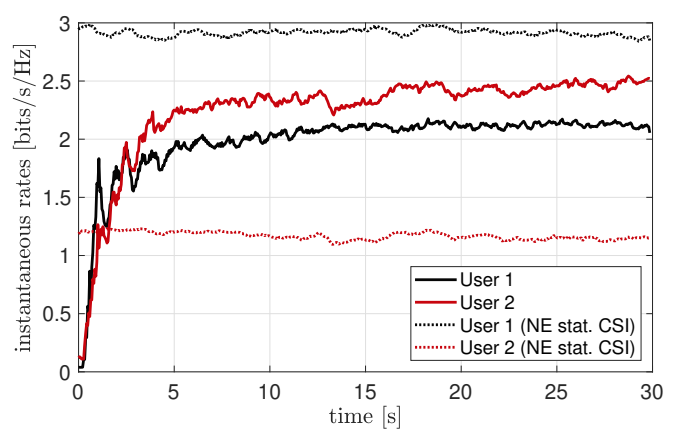

Fig. 7. Comparison between the Nash Equilibrium strategies of the game with statistical CSI and the power allocations based on instantaneous CSI in terms of "instantaneous rate" $(\beta=0.3)$.

players, they converge to a similar single-user WF allocation.

\section{CSI ACQUiSITION STRATEgIES}

\section{A. Experiment description}

In order to collect data on CSI at the transmitter, we designed an experiment with bidirectional communications. A node (called Alice) transmits and another node (called Bob) records the signal. Once Bob has finished recording, it transmits a probing signal back to Alice. Alice records this signal. Eve (the other remaining node with a hydrophone) records the signal sent by both Alice and Bob. The probing signal is 3-second-long and contains 9 repetitions of the probing maximum length sequence. A bi-directional transmission round lasts overall $12 \mathrm{~s}$, which include the signal duration, the maximum Round Trip Time in the network and technical short delays (around $4 \mathrm{~s}$ ). We repeat each bidirectional transmission round for 50 times. This phase lasts 10 minutes. This frame was repeated 3 times over the days allocated to the TNA CSIACQUA project.

\section{B. Performance Evaluation}

We quantified how 'informative' is the receive estimated channel information with respect to the transmit channel information. Among the possible metrics we considered the squared L2 norm of the standardized channel (the energy) as a function of the channel excess delay, indicated as channel length in Fig. 8.

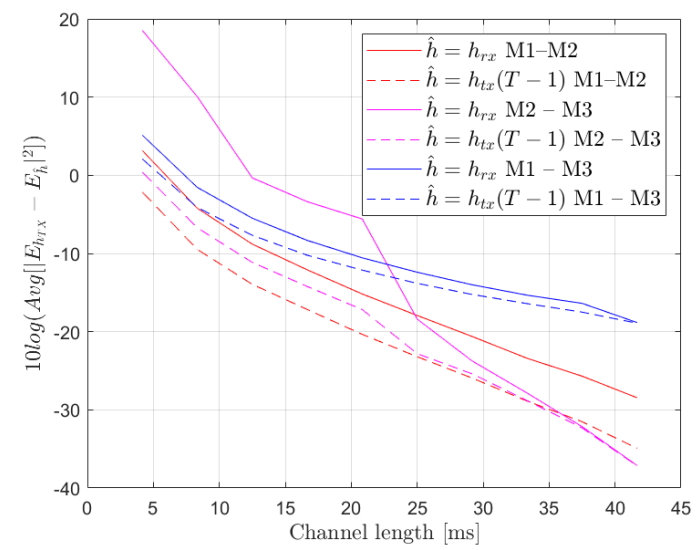

Fig. 8. MSE of the predicted and actual channel energy as a function of the channel length.

We then computed the Mean Squared Error (MSE) in dB between the energy of the transmit channel and the energy of the received channel and compared it with the MSE between the energy of the transmit channel and the energy of the transmit channel measured in the previous round (indicated as $\left.h_{t x}(T-1)\right)$. As reference, $(T-1)$ is about $5 \mathrm{~s}$. The preliminary results obtained in Fig.8. suggest that using the outdated transmit channel is never worse than using the receive channel. The channels in the two directions can have different statistics and a 'calibration' protocol could be designed to use the received channel information to infer some information on the transmit channel.

\section{CONCLUSIONS AND PERSPECTIVES}

In this work, we evaluated the benefits of adaptive power allocation strategies in underwater acoustic channels in a multiuser context, thanks to channel responses sounded at-sea. The power allocation scheme proposed here is optimal according to the Nash Equilibrium solution from game theory. This solution takes the form of a water-filling allocation, requiring the transmitters to have some CSI to adapt their transmit power on the bandwidth. We presented adaptive algorithms able to implement this solution with two types of CSI at the transmitter : an instantaneous CSI and a statistical CSI. The latter is more robust to propagation delays but harder to obtain in practice. We show that, in a strong interference context, and for both types of CSI, iterative water-filling algorithms are able to lead the channel users to an FDMA-like allocation in a totally decentralized way.

In future works, we will quantify the mutual information in terms of different metrics between the transmit channel information and the received channel information or some 
past transmit channel information in a bidirectional point-topoint UAC system. This evaluation will be used to estimate the actual performance gain obtained by an adaptive power allocation strategy that uses this not-ideal CSI. We will also give performance results in terms of outage probability instead of average (ergodic) or instantaneous rates, which can be of better practical use for systems design. The framework of stochastic games can also be of interest and capture more the several aspects of the problem that can not be properly expressed with normal-form games.

\section{ACKNOWLEDGEMENTS}

This work is supported by the European Marine Robotics (EUMR) Transnational Access (TNA) program, through the SAUWAN and CSI-ACQUA projects. It was supported by the Norwegian Research Center (NORCE) under the basic funding project number 101356 and 103892. The authors would also like to express their thanks to the team at CMRE who realized the collection of the data recorded at-sea provided for this work.

\section{REFERENCES}

[1] P. van Walree and R. Otnes, "Ultrawideband Underwater Acoustic Communication Channels," IEEE J. of Oceaninc Eng., vol. 38, no. 4, pp. 678-688, October 2013.

[2] F.-X. Socheleau, C. Laot, and J.-M. Passerieux, "Stochastic Replay of non-WSSUS Underwater Acoustic Communication Channels Recorded at Sea," IEEE Trans. Signal Process., vol. 59, no. 10, pp. 4838-4849, 2011.

[3] M. Stojanovic, S. Shahabudeen, and M. Chitre, "Underwater Acoustic Communications and Networking: Recent advances and future challenges," Marine Technology Society J., vol. 42, no. 1, 2008.

[4] J. McGee, J. Catipovic, S. Schoenecker, and P. Swaszek, "Leveraging spatial diversity to mitigate partial band interference in undersea networks through waveform reconstruction," in Proc. MTS/IEEE OCEANS 2014 - St John's, Sept 2014, pp. 1-8.

[5] Z. Wang, S. Zhou, J. Catipovic, and P. Willett, "Parameterized Cancellation of Partial-Band Partial-Block-Duration Interference for Underwater Acoustic OFDM," IEEE Trans. Signal Processing, vol. 60, no. 4, pp. 1782-1795, Apr. 2012

[6] A. Song, M. Stojanovic, and M. Chitre, "Editorial - Underwater Acoustic Communications: Where we stand and what is next?," IEEE Journal of Oceanic Engineering, vol. 44, no. 1, January.

[7] A. Pottier, F.-X. Socheleau, and C. Laot, "Robust Noncooperative Spectrum Sharing Games in Underwater Acoustic Interference Channels," IEEE J. of Oceanic Eng., vol. 42, no. 4, pp. 1019 - 1034, October 2017.

[8] A. Pottier, F.-X. Socheleau, and C. Laot, "Adaptive Power Allocation for Noncooperative OFDM Systems in UWA Interference Channels," in Proc. 2nd Underwater Acoustic Communications and Networking Conf. (UComms), Sept. 2016.

[9] A. Pottier, F.-X. Socheleau, and C. Laot, "Distributed Power Allocation Strategy in Shallow Water Acoustic Interference Channels," in IEEE Int. workshop on Signal Processing Advances in Wireless Communications (SPAWC) 2016, Jul. 2016.

[10] G. Bacci, S. Lasaulce, W. Saad, and L. Sanguinetti, "Game Theory for Networks: A tutorial on game-theoretic tools for emerging signal processing applications," IEEE Signal Processing Magazine, vol. 33, no. 1, pp. 94-119, January 2016.

[11] D. Fudenberg and D. K. Levine, The Theory of Learning in Games, Cambridge MA MIT Press, 1998.

[12] G. Scutari, D. P. Palomar, and S. Barbarossa, "Optimal Linear Precoding Strategies for Wideband Non-Cooperative Systems Based on Game Theory-Part I: Nash Equilibria," IEEE Trans. on Signal Processing, vol. 56, no. 3, pp. 1230-1249, March 2008.

[13] G. Scutari, D. P. Palomar, and S. Barbarossa, "Optimal Linear Precoding Strategies for Wideband Non-Cooperative Systems Based on Game Theory-Part II: Algorithms," IEEE Trans. on Signal Processing, vol. 56, no. 3, pp. 1250-1267, March 2008.
[14] Tevfik Yucek and HÜseyin Arslan, "Mmse noise plus interference power estimation in adaptive ofdm systems," IEEE Transactions on Vehicular Technology, vol. 56, no. 6, pp. 3857-3863, 2007.

[15] X. Lurton, An Introduction to Underwater Acoustics: Principles and Applications, Springer, 2011.

[16] Y. Aval, S. K. Wilson, and M. Stojanovic, "On the Achievable Rate of a Class of Acoustic Channels and Practical Power Allocation Strategies for OFDM Systems," IEEE J. of Oceaninc Eng., vol. 40, no. 4, pp. 785-795, Oct 2015 